\title{
HDAC6 and SIRT2 promote bladder cancer cell migration and invasion by targeting cortactin
}

\author{
QINQIN ZUO $^{1 *}$, WENJING WU $^{1 *}, \mathrm{XU} \mathrm{LI}^{1}, \mathrm{LE} \mathrm{ZHAO}^{1}$ and WEI CHEN ${ }^{1,2}$ \\ ${ }^{1}$ Center for Laboratory Medicine, and ${ }^{2}$ Clinical Laboratory, The First Affiliated Hospital, \\ School of Medicine, Xi'an Jiaotong University, Xi'an 710061, P.R. China
}

Received September 14, 2011; Accepted October 20, 2011

DOI: $10.3892 /$ or.2011.1553

\begin{abstract}
Histone deacetylase 6 (HDAC6) promotes cell motility and contributes to the metastasis of cancers. The purpose of this study was to investigate the role of HDAC6 in human bladder cancer for the first time. The results showed that HDAC6 promotes the migration and invasion of bladder cancer cells by targeting the cytoskeletal protein cortactin. Furthermore, a colony formation assay as well as in vitro migration and invasion assays demonstrated that this migration and invasion was suppressed by the HDAC6-specific inhibitor tubacin. In addition, cortactin is the substrate of SIRT2, which also belongs to the family of histone deacetylases. We demonstrated that by using SIRT2-specific siRNA combined with tubacin treatment, the cell migratory and invasive abilities were dramatically suppressed. Taken together, we conclude that HDAC6 and SIRT2 work synergistically to promote cell migration and invasion in bladder cancer, and the HDAC6-specific inhibitor tubacin may be regarded as a novel therapeutic agent for bladder cancer.
\end{abstract}

\section{Introduction}

Bladder cancer is the most common malignancy of the human urinary system in Chinese individuals, with a high rate of recurrence and a poor 5-year survival rate. The most deadly aspect of bladder cancer is its ability to metastasize. Therefore, investigating the mechanism of tumor metastasis and searching for novel therapeutic strategies have become a recent research focus.

Cell motility is associated with tumor invasiveness and metastasis. One of the key regulators involved in the cell migration process is cortactin. Cortactin, encoded by the CTTN (or EMS1) gene, is a protein originally identified as a

Correspondence to: Dr Wei Chen, Clinical Laboratory, The First Affiliated Hospital, School of Medicine, Xi'an Jiaotong University, 277 West Yanta Road, Xi'an 710061, P.R. China

E-mail: chenwei808@mail.xjtu.edu.cn

${ }^{*}$ Contributed equally

Key words: HDAC6/SIRT2, cortactin, tubacin, bladder cancer substrate of the Src tyrosine kinase (1). Its location in regions of cells undergoing membrane remodeling indicates that it may be a candidate molecule that links cell structural (cytoskeletal) organization to cell signal transduction (2). It contains a repeat region by which it interacts with F-actin to promote polymerization and branching. Accumulating evidence shows the multifunctional roles of actin-binding proteins in tumorigenic and metastatic processes of various types of human tumors (3). Cortactin is frequently overexpressed and is considered to be a prognostic marker in several types of cancers, such as breast cancer (4), hepatocellular carcinoma (5) and bladder cancer (6). Collectively, previous studies have demonstrated a strong link between cortactin expression and tumor invasiveness and metastasis. Recently, Zhang et al (7) identified it as a substrate of HDAC6 and SIRT2, two members of the histone deacetylase (HDAC) family.

HDAC6, one member of the class II HDAC family, has recently been identified as an expanded substrate such as Hsp90, tubulin and cortactin. Emerging evidence shows that HDAC6 is overexpressed in ovarian cancer cells and tissues (8) and primary oral squamous cell carcinoma cell lines (9), and is required for invadopodia formation and invasion in breast cancer cells (10). Up-regulation of HDAC6 in diverse tumors and cell lines suggests that HDAC6 is a key regulator of many signaling pathways that are linked to cancer (11). HDAC6 localizes to the motile, protrusive structures of polarized cells, including the leading edge and the uropod (12), and it participates in the formation of actin-dependent membrane structures such as ruffles and macropinosomes (13), which indicates that HDAC6 is associated with cell motility. Zhang et al (7) demonstrated that HDAC6 influences actindependent cell motility by altering the acetylation status of cortactin. Notably, they also found that a second cytoplasmic deacetylase, SIRT2, which associates with HDAC6, may also be involved in cortactin deacetylation. However, the role of HDAC6 and SIRT2 in bladder cancer cell migration and invasion remains undefined.

In the present study, we investigated the effect of HDAC6/ SIRT2 on bladder cancer cell migration and invasion. Using the HDAC6-specific inhibitor tubacin, we demonstrate that inhibition of HDAC6 caused down-regulation of both SIRT2 and cortactin, suppressed cell anchored-independent growth, colony formation ability, and prevented bladder cancer cell migration and invasion. In addition, after using a siRNA 
for SIRT2 combined with tubacin treatment, the cell migratory and invasive abilities were dramatically suppressed. Collectively, our data revealed that HDAC6 and SIRT2 cooperately promote bladder cancer cell migration and invasion through targeting cortactin.

\section{Materials and methods}

Cell culture and RNA extraction. The human bladder TCC cell line BLX-211 was cultured in RPMI-1640 medium (Gibco-BRL, Gaithersburg, MD, USA) supplemented with $10 \%$ calf serum. Cultures were maintained at $37^{\circ} \mathrm{C}$ in a humidified atmosphere with $5 \% \mathrm{CO}_{2}$. Tubacin (Sigma, USA) was prepared following the product instructions. Total RNA from the cells was extracted using TRIzol reagent (Invitrogen, USA). RNA concentration and integrity were determined by spectrophotometry and standard RNA gel electrophoresis.

Western blot analysis. The BLX-211 cells were harvested by suspension in RIPA buffer on ice for $30 \mathrm{~min}$. The protein concentrations were measured using a protein assay kit. Equal volumes of lysate were electrophoresed on a 10\% SDS polyacrylamide gel. The separated proteins in the gel were transferred to the nitrocellulose membrane. The membrane was blocked with Tris-buffered saline plus $0.1 \%$ Tween-20 (TBST) containing $10 \%$ skimmed milk overnight at $4^{\circ} \mathrm{C}$, and subsequently incubated overnight with the appropriate primary antibodies in TBST containing 5\% skimmed milk at $4^{\circ} \mathrm{C}$, followed by incubation with horseradish peroxidaseconjugated secondary immunoglobulin antibodies (Santa Cruz Biotechnology, Santa Cruz, CA, USA) for $4 \mathrm{~h}$ at room temperature. Western blot analyses were carried out using the following primary antibodies: anti-HDAC6 (Santa Cruz Biotechnology), anti-SIRT2, anti-cortactin (Millipore, USA) and anti-GAPDH (Kangcheng, Shanghai, China). Finally, the bands were visualized by chemiluminescence using a chemiluminescence kit (Pierce, USA), and the specific bands were recorded on X-ray film. GAPDH was used to normalize the protein levels.

In vitro migration and invasion assays. The invasion assay was conducted in a 24-well Millicell chamber. Pore inserts $(8-\mu \mathrm{m})$ were coated with $15 \mu \mathrm{g}$ of Matrigel (Becton-Dickinson Labware, Bedford, MA). Cells $\left(0.5 \times 10^{5}\right)$ were added to the coated filters in $100 \mu \mathrm{l}$ of serum-free medium in triplicate wells. Six hundred microliters of RPMI-1640 media containing $20 \%$ fetal bovine serum was added to the lower chamber as a chemoattractant. After $20 \mathrm{~h}$ at $37^{\circ} \mathrm{C}$ in a $5 \% \mathrm{CO}_{2}$ incubator, the Matrigel coating on the upper surface of the filter was wiped off using a cotton swab. Cells that migrated through the filters were fixed in $4 \%$ paraformaldehyde for $30 \mathrm{~min}$, stained with Giemsa, photographed and counted. The migration assay was conducted in a similar fashion without coating with Matrigel. The plates were incubated for $20 \mathrm{~h}$ then detected. Each experiment was carried out in triplicate.

Colony formation and soft agar assays. For the colony formation assay, cells were trypsinized into a single-cell suspension. A total of 100 cells were plated in each well of 6-well plates and maintained for 14 days in RPMI-1640 supplemented with $10 \%$
A

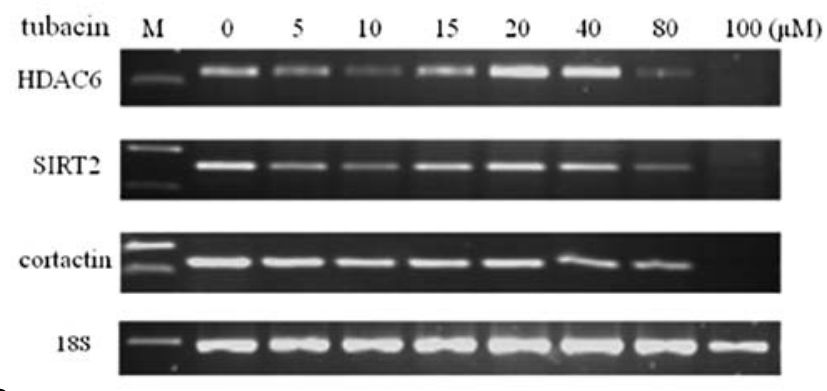

B

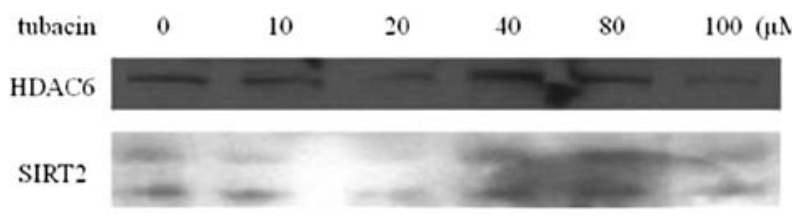

cortactin

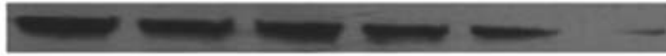

GAPDH

Figure 1. Inhibition of HDAC6 decreases the expression of both SIRT2 and cortactin in the bladder cancer cell line BLX-211. Different concentrations $(5-100 \mu \mathrm{M})$ of tubacin were used to treat BLX-211 cells. Analysis of the HDAC6, SIRT2 and cortactin expression levels was performed. (A) RT-PCR analysis shows that tubacin inhibits the expression of HDAC6, SIRT2 and cortactin at a $100 \mu \mathrm{M}$ concentration. (B) Western blot assay also shows that the expression of HDAC6, SIRT2 and cortactin was significantly suppressed by tubacin at a $100 \mu \mathrm{M}$ concentration.

calf serum to allow colony formation. Cell clones consisting of 50 cells were counted using a grid. Three independent experiments were performed.

For the soft agar assay, cells were trypsinized into a singlecell suspension. A total of 1000 cells were mixed with $1 \mathrm{ml}$ soft agar (final concentration $0.3 \%$ ) and immediately plated on top of a previously plated 1-ml layer of $0.5 \%$ agar in each well of 24-well plates. The plates were incubated in a humidified chamber with $5 \% \mathrm{CO}_{2}$ at $37^{\circ} \mathrm{C}$. After 14 days in culture, the number of clusters per plate was counted using a microscope. Groups of cells consisting of more than 50 cells per group were scored and termed 'clusters'. The colony formation ratio $=$ numbers of colonies/initiative cells $\times 100 \%$.

Wound healing assay. Cells were grown to $90 \%$ confluence in 6-well culture plates. A p200 pipet tip was used to create a scratch of the cell monolayer. Cells were washed twice with phosphate-buffered saline (PBS) in order to remove the debris from the culture, and then maintained at $37^{\circ} \mathrm{C}$ in an incubator. Images were captured immediately after wounding and 24 and $48 \mathrm{~h}$ post wounding respectively, and wound closure was monitored by microscopy. Wound sizes were verified with an ocular ruler to ensure that all wounds were the same width at the beginning of the experiment.

$R N A i$ and RT-PCR. The siRNA for SIRT2 and control were commercial products (Santa Cruz Biotechnology). BLX-211 cells were transfected using Lipofectamine 2000 reagent (Invitrogen) according to the manufacturer's instructions. 
A
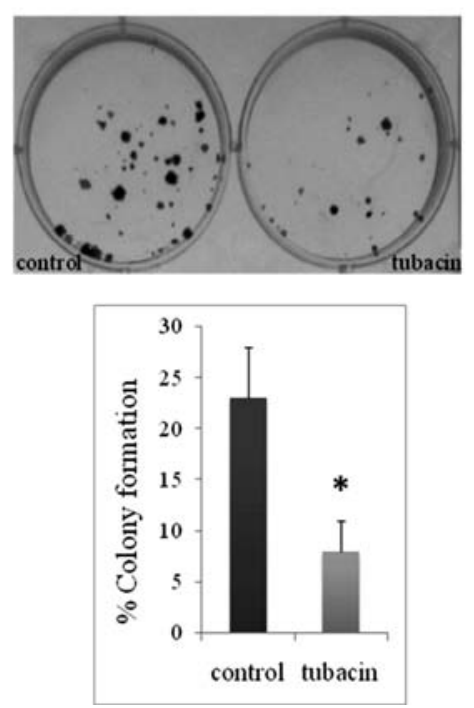

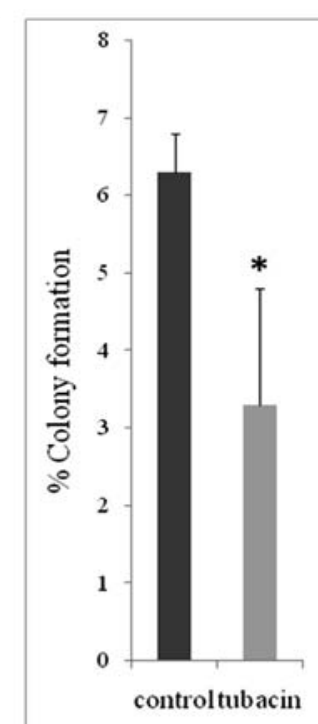

Figure 2. Inhibition of HDAC6 significantly reduces the colony formation ability of BLX-211 cells. (A) Colony formation assay. Representative cultures of tubacin-treated cells (right well) compared to control cells (left well) are shown (magnification, $\mathrm{x} 10$ ). Values represent the means $\pm \mathrm{SD}$ of at least three independent experiments. ${ }^{~} \mathrm{P}<0.05$ indicates that the colony formation was significantly reduced by the suppression of HDAC6. (B) Soft agar assay. Values represent the means \pm SD of at least three independent experiments ${ }^{*} \mathrm{P}<0.05$ indicates that the anchorage-independent growth of the cells was significantly inhibited by the suppression of HDAC6.

Total RNA was extracted 24 and $48 \mathrm{~h}$ after transfection. cDNA synthesis was conducted using the PrimeScript First Strand cDNA Synthesis Kit (Takara, Japan) according to the manufacturer's instructions. The primer sequences for PCR are listed as follows: HDAC6 (NM_006044) forward primer, 5'-CATCCGAACTCATACTCCTGTGC-3'; reverse primer, 5'-CAATAGCCATCCATAAGACTGTGC-3'. SIRT2 (NM_000593) forward primer, 5'-TCTGAGGT GACGCCCAAGTGT-3'; reverse primer, 5'-TGCTGA TGAGGGAGGCAAAGG-3'. Cortactin (NM_005231) forward primer, 5'-TGTGGAACAAGACCGAATGGA-3'; reverse primer, 5'-TGGTATTCAAAGCCTACAGCAGA-3'. 18S rRNA (M10098) forward primer, 5'-CAGCCACC CGAGATTGAGCA-3'; reverse primer, 5'-TAGTAGCGA CGGGCGGTGTG-3'

Statistical analysis. All statistical analyses were performed using the SPSS13.0 software. The results were expressed as mean \pm standard deviation (SD) of at least three separate experiments. The differences between groups were analyzed using the Student's t-test. A difference was deemed statistically significant at $\mathrm{P}<0.05$.

\section{Results}

Inhibition of HDAC6 decreases the expression of both SIRT2 and cortactin in a bladder cancer cell line. In order to assess the regulation of HDAC6 to SIRT2 and cortactin in bladder cancer cells, we first evaluated the mRNA and protein expression of HDAC6, SIRT2 and cortactin in the BLX-211 bladder cancer cell line. Reverse transcription PCR and Western blot

A

$0 \mathrm{~h}$

$24 \mathrm{~h}$
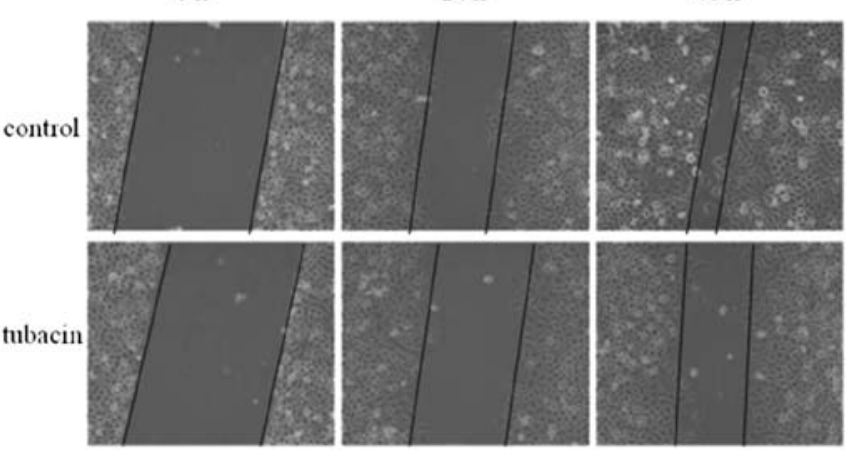

B
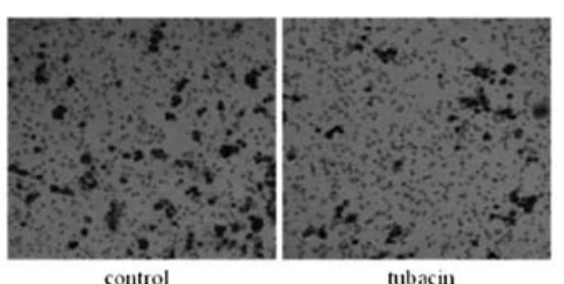

tubacin
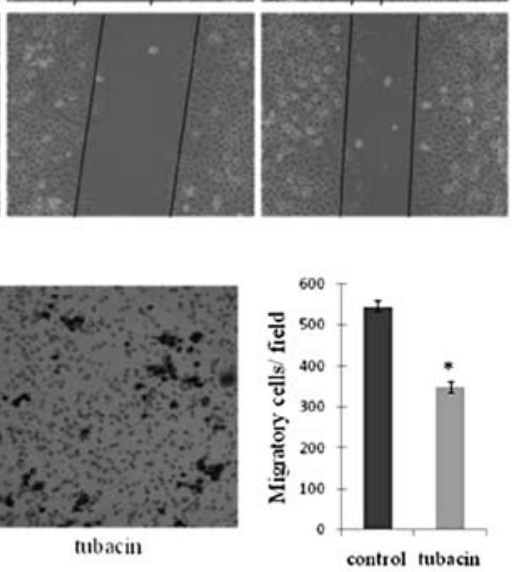

C
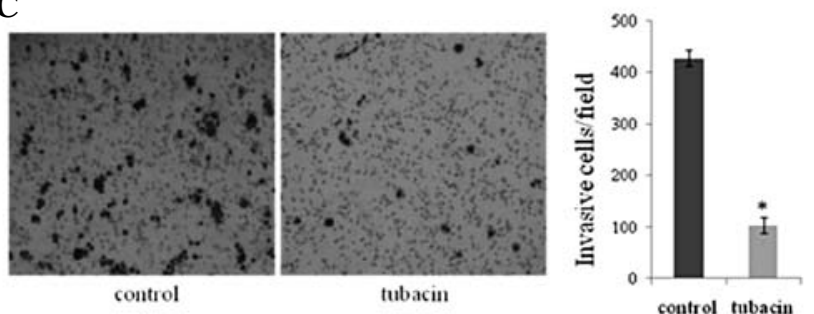

Figure 3. HDAC6 suppression prevents cell motility. (A) Wound healing assay shows that the suppression of HDAC6 resultes in decreased motility of BLX-211 cells. The images are representative of at least three independen experiments (magnification, $\mathrm{x} 10$ ). ( $\mathrm{B}$ and $\mathrm{C}$ ) In vitro migration and invasion assays. Representative number of motile or invading cells was counted under a microscope in five random fields at $\mathrm{x} 200$. Tubacin-treated cells showed less motile and invasive abilities than control cells $\left({ }^{*} \mathrm{P}<0.05\right)$.

analysis were performed. The results showed that HDAC6, SIRT2 and cortactin were expressed in the BLX-211 cells. The cells were then treated with the HDAC6-specific inhibitor tubacin using different concentrations from 5 to $100 \mu \mathrm{M}$. The results showed that when the cells were treated with $100 \mu \mathrm{M}$ tubacin, the mRNA levels of HDAC6, SIRT2 and cortactin were significantly decreased (Fig. 1A). These changes also occured at the protein level as determined by Western blot analysis (Fig. 1B). The concentration of $100 \mu \mathrm{M}$ was chosen to carry out the following studies. These data indicate that HDAC6 may contribute to the regulation of both SIRT2 and cortactin.

Effect of suppression of HDAC6 on BLX-211 cells in vitro. The effect of HDAC6 suppression on the cell characteristics of BLX-211 cells was tested by several assays. The proliferative ability and anchorage-independent growth of the cells were assessed by colony formation and soft agar assays. The numbers of colonies produced by the tubacin-treated cells were greatly reduced by $65.2 \%(\mathrm{P}<0.05)$ (Fig. $2 \mathrm{~A})$ and $50.0 \%$ $(\mathrm{P}<0.05)$ (Fig. 2B), respectively, in comparison with the number that developed in the control cells. These results suggest that inhibition of HDAC6 by tubacin impairs the cell viability and anchorage-independent growth of BLX-211 cells in vitro. 
A

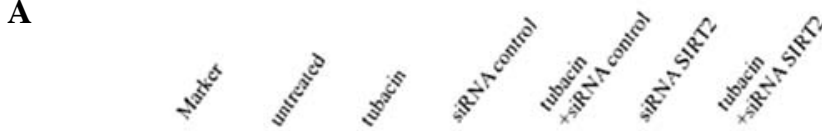

HDAC6

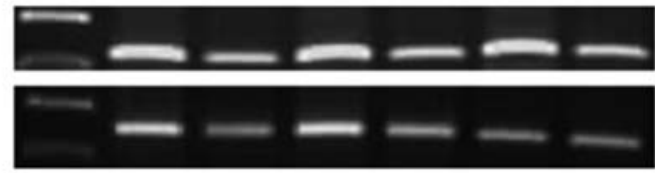

SIRT2

cortactin

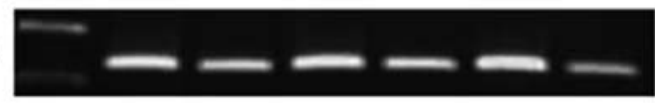

$18 \mathrm{~S}$

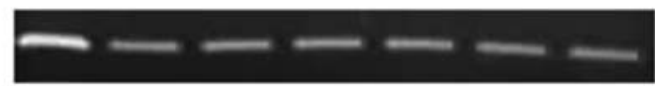

B

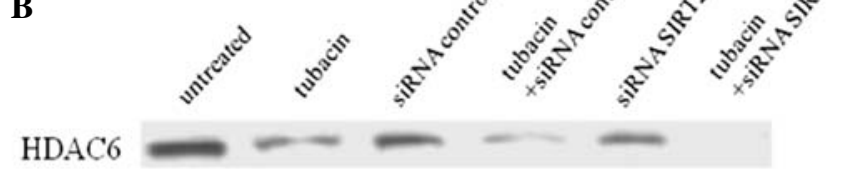

SIRT2

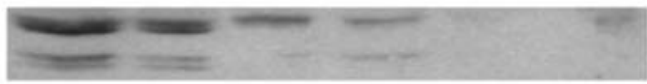

cortactin

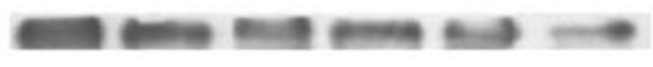

GAPDH

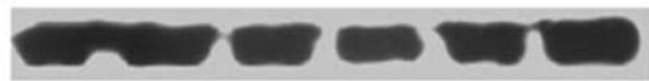

Figure 4. Tubacin treatment combined with SIRT2 knockdown resulted in the further down-regulation of cortactin. RT-PCR analysis (A) and Western blot assay (B) show that the transfection of siRNA decreased both the SIRT2 mRNA and protein expression without affecting HDAC6 expression. In the SIRT2-knockdown cells, no decreased mRNA expression of cortactin was observed while the protein level was slightly reduced. In the tubacin-treated combined with SIRT2-knockdown cells, both mRNA and protein expression levels of cortactin were significantly reduced.

Moreover, the wound healing assay was utilized to observe the effect of HDAC6 suppression on cell motility. The suppression of HDAC6 resulted in attenuated motility of BLX-211 cells. The wound healing was significantly delayed in the tubacin-treated BLX-211 cells (Fig. 3A).

Furthermore, the migration and invasiveness of different cells were assessed using the migration and invasion assays as described in Materials and methods. As shown in Fig. 3B and $\mathrm{C}$, the abilities of cell migration and invasion were significantly reduced by $36.0 \%(\mathrm{P}<0.05)$ and $75.9 \%(\mathrm{P}<0.05)$ after HDAC6 inhibition. Thus, suppression of HDAC6 expression notably reduces the migration and invasiveness of bladder cancer cells.

Tubacin treatment combined with SIRT2 knockdown results in further down-regulation of cortactin. To investigate the role of tubacin treatment combined with SIRT2 knockdown in the BLX-211 cells, we transfected SIRT2 siRNA into BLX-211 cells while treating cells with tubacin at the same time. As shown in Fig. 4, transfection of siRNA decreased both the SIRT2 mRNA and protein expression without affecting HDAC6 expression. In the SIRT2-knockdown cells, no decreased mRNA expression of cortactin was observed while the protein level was slightly reduced. In the tubacintreated combined with SIRT2-knockdown cells, both mRNA
A
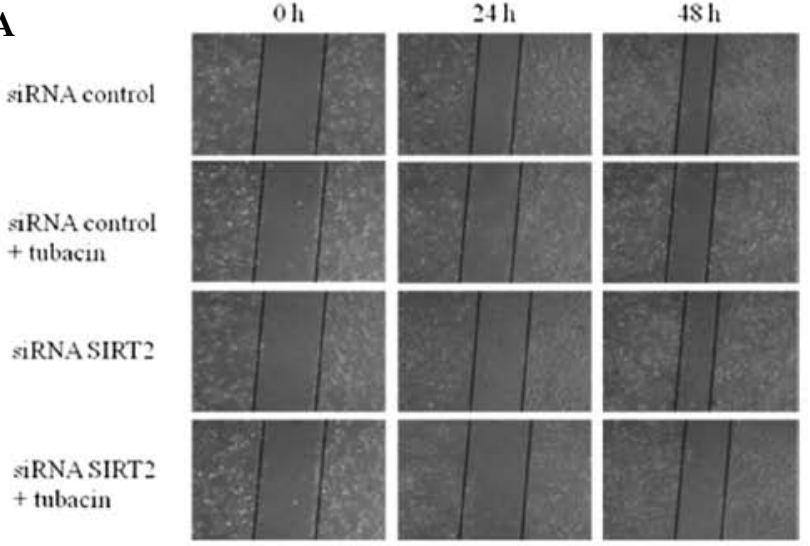

B
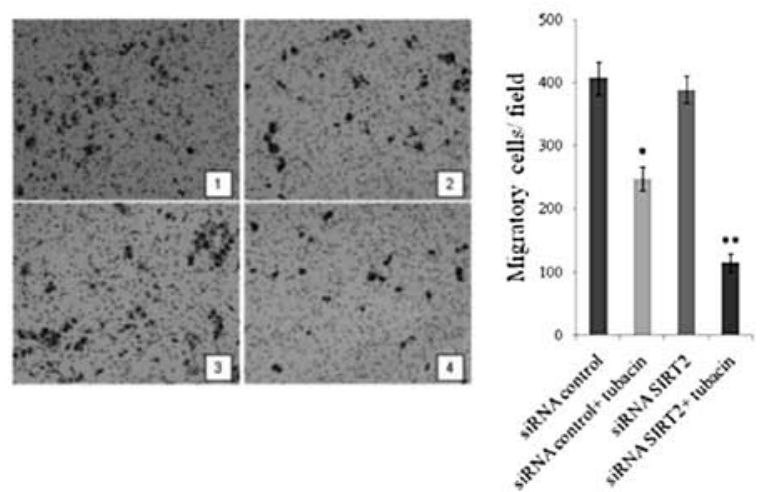

C
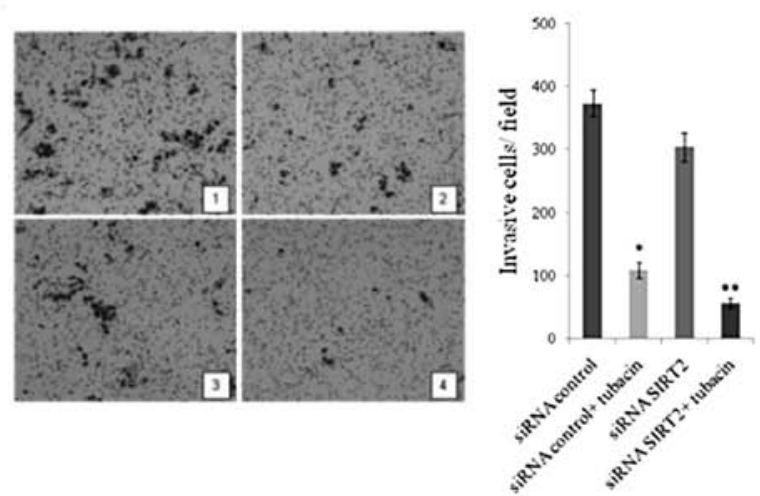

Figure 5. Effect of tubacin treatment combined with SIRT2 knockdown on cell migration and invasiveness in vitro. (A) Wound healing assay. Cell motility was not significantly decreased when SIRT2 expression was inhibited by siRNA. Yet, when the cells were treated with tubacin together with SIRT2 siRNA, the recovery of the wound was significantly delayed. The representative images were captured immediately after wounding and 24 and $48 \mathrm{~h}$ post wounding. (B and C) In vitro migration and invasion assays. Tubacin treatment combined with SIRT2 siRNA resulted in further inhibition of cell migration and invasion compared to the untreated control cells. Representative number of migrating or invading cells was counted under the microscope in five random fields at x200. 1, siRNA control; 2 , siRNA control + tubacin; 3, siRNA SIRT2; 4, siRNA SIRT2 + tubacin. $\left({ }^{*} \mathrm{P}<0.05,{ }^{* *} \mathrm{P}<0.01\right)$.

and protein expression levels of cortactin were detected to be significantly reduced.

Effect of tubacin treatment combined with SIRT2 knockdown on cell migration and invasiveness in vitro. The wound healing assay showed that the cell motility was not significantly decreased when SIRT2 expression was inhibited by siRNA. 
Meanwhile, we found that when the cells were treated with tubacin together with SIRT2 siRNA, the wound recovery was significantly delayed (Fig. 5A).

Similar to the wound healing assay results, the migration and invasive assays of different cells (Fig. 5B and C) showed that treatment with SIRT2 siRNA alone did not affect the cell migration and invasive abilities, compared to the control cells. When the cells were treated with tubacin together with SIRT2 siRNA, the abilities of cell migration and invasion were dramatically reduced by $72 \%(\mathrm{P}<0.05)$ and $85 \%(\mathrm{P}<0.05)$. Together, these data indicate that HDAC6 and SIRT2 work cooperatively to promote bladder cancer cell migration and invasion by targeting cortactin.

\section{Discussion}

In the present study, utilizing the HDAC6-specific inhibitor tubacin and RNAi technique, we demonstrated that HDAC6 works cooperately with SIRT2 to promote bladder cancer cell migration and invasion by targeting cortactin. Therefore, this study may provide one possible mechanism of invasion and metastasis in bladder cancer, and further investigation of HDAC6 and SIRT2 as therapeutic targets against bladder cancer invasion and metastasis is warranted.

As a cytoplasmic enzyme, HDAC6 is a key regulator in many pivotal biological processes, including cell migration, immune synapse formation, viral infection, and the degradation of misfolded proteins (14). To date, mounting evidence links HDAC6 to cell migration in at least two different manners: a tubulin-deacetylase- and/or cortactin-deacetylasedependent manner, in the case of the non-chemotactic migration of fibroblasts, and independently of its deacetylase activity for chemokine-induced lymphocyte migration (14). In this study, we demonstrated that when the bladder cancer cells were treated with the HDAC6-specific inhibitor tubacin, the expression of HDAC6 and SIRT2 was down-regulated, and the expression of cortactin, a known HDAC6 and SIRT2 deacetylated substrate, was decreased as well. As a result, the cell migration and invasiveness were both inhibited. This may be an underlying mechanism for explaining the invasion and metastasis in bladder cancer.

Meanwhile, the study of Zhang et al indicated that SIRT2 regulates both microtubules and actin cytoskeletons associated with HDAC6 (7). In our study, we also investigated the role of SIRT2 in bladder cancer cell migration. After knockdown of SIRT2 using siRNA, the expression of HDAC6 was not affected, while the expression of cortactin was slightly decreased. However, we did not observe a significant impact on cell migration and invasion when the cells were only treated with SIRT2-siRNA. But when the cells were treated by tubacin combined with SIRT2-siRNA, the cell migration and invasion were dramatically delayed more than that in the tubacin-only or the siRNA-only group. These results indicated that HDAC6 cooperates with SIRT2 to down-regulate cortactin and inhibit the cell motility further.

HDAC inhibitors have been proposed for the treatment of cancer (15). Histone hyperacetylation induced by HDAC inhibitors such as TSA correlates with gene expression, cell cycle arrest, cell differentiation and cell death (16). Another small-molecule inhibitor is tubacin, which selectively inhibits
HDAC6 and causes increased acetylation of $\alpha$-tubulin, accumulation of polyubiquitinated proteins and apoptosis $(16,17)$. However, it was not known to alter the expression of HDAC6. In our study, the PCR and Western blot results showed that both the expression levels of HDAC6 RNA and protein were suppressed by treatment of tubacin at $100 \mu \mathrm{M}$. We speculated that the reason was due to the higher concentration used. A higher concentration of tubacin may not only inhibit the deacetylase activity but also regulate the expression of HDAC6. Additionally, the change in HDAC6 expression resulted in corresponding changes in cortactin expression. In support of our view, tubacin was able to induce angiostatic ADAMTS1 expression by the specific inhibition of HDAC6 in A549 cells (18).

Furthermore, HDAC6 contains two homologous catalytic domains. In one previous study, both HDAC6 catalytic domains were required for full tubulin deacetylase (TDAC) activity $(19,20)$; however, in other studies the TDAC activity was attributed to the second domain $(16,21,22)$. Tubacin binds only to the catalytic domain for tubulin deacetylation but not to that for histone deacetylation of HDAC6. Notably, cortactin also binds to the second catalytic domain of HDAC6 (7). Since the role of HDAC6 and SIRT2 in cell migration is not limited to microtubule deacetylation but also involves the actin cytoskeleton, studies using drugs which inhibit HDAC6 now need to account for their effects on both cytoskeletal elements (23). Thus, whether tubacin affects the deacetylation of cortactin needs to be further investigated.

Taken together, our data showed for the first time that HDAC6, associated with SIRT2, promotes the migration and invasion of bladder cancer cells by targeting cortactin and these abilities are suppressed by the HDAC6-specific inhibitor tubacin. Thus, tubacin could be considered as a possible therapeutic agent for inhibiting the invasion and metastasis of bladder cancer. Further studies in vivo are required to assess the potential of tubacin in the treatment of cancer.

\section{Acknowledgements}

Wenjing Wu was supported by an Exchange Scholarship from the Kwang-Hua Education Foundation of Xi'an Jiaotong Unversity.

\section{References}

1. Wu H, Reynolds AB, Kanner SB, Vines RR and Parsons JT: Identification and characterization of a novel cytoskeletonassociated pp60src substrate. Mol Cell Biol 11: 5113-5124, 1991.

2. Cosen-Binker LI and Kapus A: Cortactin: the gray eminence of the cytoskeleton. Physiology (Bethesda) 21: 352-361, 2006.

3. Yamaguchi $\mathrm{H}$ and Condeelis J: Regulation of the actin cytoskeleton in cancer cell migration and invasion. Biochim Biophys Acta 1773: 642-652, 2007.

4. Ormandy CJ, Musgrove EA, Hui R, Daly RJ and Sutherland RL: Cyclin D1, EMS1 and 11q13 amplification in breast cancer. Breast Cancer Res Treat 78: 323-335, 2003.

5. Yuan BZ, Zhou X, Zimonjic DB, Durkin ME and Popescu NC: Amplification and overexpression of the EMS 1 oncogene, a possible prognostic marker, in human hepatocellular carcinoma. J Mol Diagn 5: 48-53, 2003

6. Zaharieva BM, Simon R, Diener PA, et al: High-throughput tissue microarray analysis of 11q13 gene amplification (CCND1, FGF3, FGF4, EMS1) in urinary bladder cancer. J Pathol 201: 603-608, 2003. 
7. Zhang X, Yuan Z, Zhang Y, et al: HDAC6 modulates cell motility by altering the acetylation level of cortactin. Mol Cell 27: 197-213, 2007

8. Bazzaro M, Lin Z, Santillan A, et al: Ubiquitin proteasome system stress underlies synergistic killing of ovarian cancer cells by bortezomib and a novel HDAC6 inhibitor. Clin Cancer Res 14: 7340-7347, 2008.

9. Sakuma T, Uzawa K, Onda T, et al: Aberrant expression of histone deacetylase 6 in oral squamous cell carcinoma. Int J Oncol 29: 117-124, 2006

10. Rey M, Irondelle M, Waharte F, Lizarraga F and Chavrier P: HDAC6 is required for invadopodia activity and invasion by breast tumor cells. Eur J Cell Biol 90: 128-135, 2011.

11. Aldana-Masangkay GI and Sakamoto KM: The role of HDAC6 in cancer. J Biomed Biotechnol 2011: 875824, 2011.

12. Cabrero JR, Serrador JM, Barreiro O, et al: Lymphocyte chemotaxis is regulated by histone deacetylase 6 , independently of its deacetylase activity. Mol Biol Cell 17: 3435-3445, 2006.

13. Gao YS, Hubbert CC, Lu J, Lee YS, Lee JY and Yao TP: Histone deacetylase 6 regulates growth factor-induced actin remodeling and endocytosis. Mol Cell Biol 27: 8637-8647, 2007.

14. Valenzuela-Fernandez A, Cabrero JR, Serrador JM and SanchezMadrid F: HDAC6: a key regulator of cytoskeleton, cell migration and cell-cell interactions. Trends Cell Biol 18: 291-297, 2008.

15. Lane AA and Chabner BA: Histone deacetylase inhibitors in cancer therapy. J Clin Oncol 27: 5459-5468, 2009.
16. Haggarty SJ, Koeller KM, Wong JC, Grozinger CM and Schreiber SL: Domain-selective small-molecule inhibitor of histone deacetylase 6 (HDAC6)-mediated tubulin deacetylation. Proc Natl Acad Sci USA 100: 4389-4394, 2003.

17. Namdar M, Perez G, Ngo L and Marks PA: Selective inhibition of histone deacetylase 6 (HDAC6) induces DNA damage and sensitizes transformed cells to anticancer agents. Proc Natl Acad Sci USA 107: 20003-20008, 2010.

18. Chou CW and Chen CC: HDAC inhibition upregulates the expression of angiostatic ADAMTS1. FEBS Lett 582: 4059-4065, 2008.

19. Zhang Y, Li N, Caron C, et al: HDAC-6 interacts with and deacetylates tubulin and microtubules in vivo. EMBO $\mathrm{J} 22$ : 1168-1179, 2003.

20. Zhang Y, Gilquin B, Khochbin S and Matthias P: Two catalytic domains are required for protein deacetylation. J Biol Chem 281: 2401-2404, 2006.

21. Matsuyama A, Shimazu T, Sumida Y, et al: In vivo destabilization of dynamic microtubules by HDAC6-mediated deacetylation. EMBO J 21: 6820-6831, 2002.

22. Zou H, Wu Y, Navre M and Sang BC: Characterization of the two catalytic domains in histone deacetylase 6 . Biochem Biophys Res Commun 341: 45-50, 2006.

23. Luxton GW and Gundersen GG: HDAC6-pack: cortactin acetylation joins the brew. Dev Cell 13: 161-162, 2007. 